\title{
KAZHDAN-LUSZTIG BASIS AND A GEOMETRIC FILTRATION OF AN AFFINE HECKE ALGEBRA
}

\author{
TOSHIYUKI TANISAKI AND NANHUA XI
}

\section{Dedicated to Professor George Lusztig on his sixtieth birthday}

\begin{abstract}
According to Kazhdan-Lusztig and Ginzburg, the Hecke algebra of an affine Weyl group is identified with the equivariant $K$-group of Steinberg's triple variety. The $K$-group is equipped with a filtration indexed by closed $G$-stable subvarieties of the nilpotent variety, where $G$ is the corresponding reductive algebraic group over $\mathbb{C}$. In this paper we will show in the case of type $A$ that the filtration is compatible with the Kazhdan-Lusztig basis of the Hecke algebra.
\end{abstract}

\section{$\S 0$. Introduction}

Let $G$ be a connected reductive algebraic group over the complex number field $\mathbb{C}$ with simply-connected derived group. Let $W$ and $P$ be its Weyl group and weight lattice respectively. The semidirect product $\tilde{W}_{a}=W P$ with respect to the action of $W$ on $P$ is called an (extended) affine Weyl group. Let $H\left(\tilde{W}_{a}\right)$ be the associated Hecke algebra. According to KazhdanLusztig and Ginzburg ([6], [3]) we have a geometric realization of $H\left(\tilde{W}_{a}\right)$ in terms of equivariant $K$-theory. Namely, we have an isomorphism

$$
\Phi: H\left(\tilde{W}_{a}\right) \longrightarrow K^{G \times \mathbb{C}^{*}}(Z)
$$

of $\mathbb{Z}\left[q^{1 / 2}, q^{-1 / 2}\right]$-algebras, where $K^{G \times \mathbb{C}^{*}}(Z)$ denotes the equivariant $K$-group of Steinberg's triple variety $Z$ with respect to the natural action of $G \times \mathbb{C}^{*}$. Let $\mathcal{N}$ be the nilpotent variety of the Lie algebra $\mathfrak{g}$ of $G$. For each $G$-stable closed subset $V$ of $\mathcal{N}$ there corresponds a $G \times \mathbb{C}^{*}$-stable closed subvariety $Z_{V}$ of $Z$, and the associated equivariant $K$-group $K^{G \times \mathbb{C}^{*}}\left(Z_{V}\right)$ is identified with a two-sided ideal of $K^{G \times \mathbb{C}^{*}}(Z)$. Moreover, we have $K^{G \times \mathbb{C}^{*}}\left(Z_{V_{1}}\right) \subset$ $K^{G \times \mathbb{C}^{*}}\left(Z_{V_{2}}\right)$ if $V_{1} \subset V_{2}$.

Received April 27, 2005.

2000 Mathematics Subject Classification: 20G05, 18F25, 20 C08.

$\mathrm{N}$. Xi was partially supported by a fund of 973 program. 
Recall that $H\left(\tilde{W}_{a}\right)$ is equipped with the Kazhdan-Lusztig basis $\left\{C_{w} \mid\right.$ $\left.w \in \tilde{W}_{a}\right\}([5])$. It plays very important roles in various aspects of the representation theory of reductive algebraic groups. It should be an interesting problem to give a geometric description of $\Phi\left(C_{w}\right)$ for $w \in \tilde{W}_{a}$. An answer in the case $w \in W$ is given in [18]. Moreover, the answer for certain elements corresponding to dominant elements in $P$ is given in [13]. Related to this problem, it is conjectured that $K^{G \times \mathbb{C}^{*}}\left(Z_{V}\right)$ is spanned by a subset of $\left\{\Phi\left(C_{w}\right) \mid w \in \tilde{W}_{a}\right\}$ for any $G$-stable closed subset $V$ of $\mathcal{N}$. In particular, any $H\left(\tilde{W}_{a}\right)$-bimodule associated to a two-sided cell of $\tilde{W}_{a}$ should be identified with $K^{G \times \mathbb{C}^{*}}\left(Z_{\bar{O}}\right) / K^{G \times \mathbb{C}^{*}}\left(Z_{\bar{O} \backslash O}\right)$ for a nilpotent orbit $O$.

The aim of this paper is to prove this conjecture in the case $G$ is of type $A$. A key to this result is the fact that the $H\left(\tilde{W}_{a}\right)$-bimodule corresponding to a two-sided cell of $\tilde{W}_{a}$ is generated by a single element (see Theorem 4.3 below).

The contents of this paper are as follows. In Section 1 and Section 2 we will recall some fundamental facts on (affine) Hecke algebras. A precise formulation of the above stated conjecture in view of the bijection between the set of nilpotent orbits and that of two-sided cells will be given in Section 3 . In Section 4 we will give a proof of the conjecture in the case $G=G L_{n}(\mathbb{C})$. The arguments works for $S L_{n}(\mathbb{C})$ as well. In Appendix A we will collect well-known facts on equivariant $K$-theory, and in Appendix B we will give a description of the product on the quotient $K^{G \times \mathbb{C}^{*}}\left(Z_{\bar{O}}\right) / K^{G \times \mathbb{C}^{*}}\left(Z_{\bar{O} \backslash O}\right)$ for any $G$ in terms of the Springer fiber and Slodowy's variety, where $O$ is a nilpotent orbit.

\section{§1. Hecke algebras}

Let $(W, S)$ be a Coxeter system with the length function $\ell: W \rightarrow \mathbb{Z}_{\geq 0}$ and the standard partial order $\geq$. Assume that we are given a group $\Omega$ and a group homomorphism $\Omega \rightarrow \operatorname{Aut}(W, S)$, where $\operatorname{Aut}(W, S)$ denotes the automorphism group of $(W, S)$. We denote by $\tilde{W}$ the semidirect product $\Omega W$ with respect to the action of $\Omega$ on $W$. The length function $\ell$ and the standard partial order $\geq$ for $W$ are naturally extended to $\tilde{W}$ by

$$
\begin{aligned}
& \ell(\omega w)=\ell(w) \quad(\omega \in \Omega, w \in W), \\
& \omega_{1} w_{1} \geq \omega_{2} w_{2} \Longleftrightarrow \omega_{1}=\omega_{2}, w_{1} \geq w_{2} \quad\left(\omega_{1}, \omega_{2} \in \Omega, w_{1}, w_{2} \in W\right) .
\end{aligned}
$$

For $w$ in $\tilde{W}$ we set

$$
L(w)=\{s \in S \mid s w \leq w\}, \quad R(w)=\{s \in S \mid w s \leq w\} .
$$


We denote by $H(\tilde{W})$ the Hecke algebra associated to $\tilde{W}$. It is an associative algebra over the Laurent polynomial ring $\mathbb{Z}\left[q^{1 / 2}, q^{-1 / 2}\right]$. As a $\mathbb{Z}\left[q^{1 / 2}, q^{-1 / 2}\right]$-module it has a free basis $\left\{T_{w} \mid w \in \tilde{W}\right\}$, and the multiplication is determined by

$$
\begin{aligned}
& T_{y} T_{w}=T_{y w} \quad(y, w \in \tilde{W}, \ell(y)+\ell(w)=\ell(y w)) \\
& \left(T_{s}+1\right)\left(T_{s}-q\right)=0 \quad(s \in S) .
\end{aligned}
$$

There is a unique ring automorphism $h \mapsto \bar{h}$ of $H(\tilde{W})$ determined by

$$
\overline{q^{1 / 2}}=q^{-1 / 2}, \quad \bar{T}_{w}=T_{w^{-1}}^{-1} \quad(w \in \tilde{W}) .
$$

According to Kazhdan-Lusztig [5], for each $w \in \tilde{W}$ there exists uniquely an element

$$
C_{w}=\sum_{y \leq w} P_{y, w}(q) T_{y}
$$

of $H(\tilde{W})$ satisfying

(a) $P_{w, w}(q)=1$,

(b) for $y<w$ we have $P_{y, w}(q) \in \mathbb{Z}[q]$, and $\operatorname{deg}\left(P_{y, w}(q)\right) \leq(\ell(w)-\ell(y)-$ 1) $/ 2$,

(c) $\bar{C}_{w}=q^{-\ell(w)} C_{w}$.

The basis $\left\{C_{w} \mid w \in \tilde{W}\right\}$ of $H(\tilde{W})$ is called the Kazhdan-Lusztig basis. We will also use

$$
C_{w}^{\prime}=q^{-\ell(w) / 2} C_{w} \quad(w \in \tilde{W}) .
$$

For $w \in \tilde{W}$ let $\mathcal{I}_{w}\left(\right.$ resp. $\left.\mathcal{I}_{w}^{L}, \mathcal{I}_{w}^{R}\right)$ denote the set of two-sided (resp. left, right) ideals $I$ of $H(\tilde{W})$ subject to the conditions

(a) $C_{w} \in I$,

(b) $I$ is spanned over $\mathbb{Z}\left[q^{1 / 2}, q^{-1 / 2}\right]$ by a subset of $\left\{C_{y} \mid y \in \tilde{W}\right\}$.

It contains the unique minimal element $I_{w}=\bigcap_{I \in \mathcal{I}_{w}} I$ (resp. $I_{w}^{L}=\bigcap_{I \in \mathcal{I}_{w}^{L}} I$, $\left.I_{w}^{R}=\bigcap_{I \in \mathcal{I}_{w}^{R}} I\right)$. We define a preorder $\underset{L R}{\leq}($ resp. $\underset{L}{\leq}, \underset{R}{\leq})$ and an equivalence 
relation $\underset{L R}{\sim}(\operatorname{resp} . \underset{L}{\sim}, \underset{R}{\sim})$ on $\tilde{W}$ by

$$
\begin{aligned}
& y \underset{L R}{\leq} w \Longleftrightarrow I_{y} \subset I_{w}, \\
& \text { (resp. } \left.y \underset{L}{\leq} w \Longleftrightarrow I_{y}^{L} \subset I_{w}^{L}, y \underset{R}{\leq} w \Longleftrightarrow I_{y}^{R} \subset I_{w}^{R}\right) \\
& y \underset{L R}{\sim} w \Longleftrightarrow I_{y}=I_{w}, \\
& \left(\operatorname{resp.} y \underset{L}{\sim} w \Longleftrightarrow I_{y}^{L}=I_{w}^{L}, y \underset{R}{\sim} w \Longleftrightarrow I_{y}^{R}=I_{w}^{R}\right) .
\end{aligned}
$$

Equivalence classes with respect to $\underset{L R}{\sim}(\operatorname{resp} . \underset{L}{\sim}, \underset{R}{\sim})$ are called two-sided (resp. left, right) cells of $\tilde{W}$. The preorder $\underset{L R}{\leq}$ on $\tilde{W}$ induces a partial order on the set of two-sided cells which is also denoted by $\underset{L R}{\leq}$. For a two-sided cell $\mathcal{C}$ of $\tilde{W}$ with $w \in \mathcal{C}$ we define two-sided ideals $H(\tilde{W})_{L R}^{\leq \mathcal{C}}$ and $H(\tilde{W})_{L R}^{<\mathcal{C}}$ of $H(\tilde{W})$ by

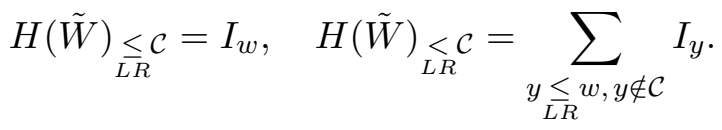

The $H(\tilde{W})$-bimodule

$$
H(\tilde{W})_{\mathcal{C}}=H(\tilde{W})_{L R}^{\leq \mathcal{C}} / H(\tilde{W})_{L R}^{<\mathcal{C}}
$$

has a canonical $\mathbb{Z}\left[q^{1 / 2}, q^{-1 / 2}\right]$-basis parametrized by $\mathcal{C}$. The multiplication of $H(\tilde{W})$ induces a multiplication of $H(\tilde{W})_{\mathcal{C}}$ which is associative; however, $H(\tilde{W})_{\mathcal{C}}$ does not contain the identity element in general.

Lemma 1.1. (Kazhdan-Lusztig [5]) If $y \leq \frac{\leq}{L} w$ (resp. $y \leq \frac{\leq}{R} w$ ), then $R(w) \subset R(y)$ (resp. $L(w) \subset L(y))$. In particular, if $y \underset{L}{\sim} w($ resp. $y \underset{R}{\sim} w)$, then $R(w)=R(y)$ (resp. $L(w)=L(y))$.

For a subset $T$ of $S$ such that $\langle T\rangle$ is a finite subgroup of $W$ we denote the longest element of $\langle T\rangle$ by $w_{T}$. We call $w \in \tilde{W}$ a parabolic element if there exists some $T \subset S$ such that $|\langle T\rangle|<\infty$ and $w=w_{T}$.

We will need the following simple assertion later.

LEMma 1.2. Let $x, y \in \tilde{W}$ and let $w$ be a parabolic element of $\tilde{W}$. Assume that $x \leq \frac{L}{L} w$ and $y \leq w$. Then $C_{x}^{\prime}=h C_{w}^{\prime}$ and $C_{y}^{\prime}=C_{w}^{\prime} h^{\prime}$ for some $h, h^{\prime} \in H(\tilde{W})$. 
Proof. By Lemma 1.1 we have $R(w) \subset R(x)$ and $L(w) \subset L(y)$. Since $w$ is a parabolic element, there are $x_{1}$ and $y_{1}$ in $\tilde{W}$ such that $x=x_{1} w$, $y=w y_{1}$ and $\ell(x)=\ell\left(x_{1}\right)+\ell(w), \ell(y)=\ell(w)+\ell\left(y_{1}\right)$. Now using induction on the length of $x_{1}$ and of $y_{1}$ we see the assertion is true (see [5, (2.3.a), (2.3.b)]).

In the analysis of two-sided cells the star operations defined in KazhdanLusztig [5] and the $a$-function defined in Lusztig [10] play important roles.

Let $s$ and $t$ be in $S$ such that $s t$ has order 3, i.e. sts $=t s t$. Define

$$
\begin{aligned}
& D_{L}(s, t)=\{w \in \tilde{W} \mid L(w) \cap\{s, t\} \text { has exactly one element }\}, \\
& D_{R}(s, t)=\{w \in \tilde{W} \mid R(w) \cap\{s, t\} \text { has exactly one element }\} .
\end{aligned}
$$

If $w$ is in $D_{L}(s, t)$, then $\{s w, t w\}$ contains exactly one element in $D_{L}(s, t)$, denoted by ${ }^{*} w$, here $*=\{s, t\}$. The map

$$
D_{L}(s, t) \ni w \longmapsto{ }^{*} w \in D_{L}(s, t)
$$

is an involution and is called a left star operation. Similarly we can define the right star operation $D_{R}(s, t) \ni w \mapsto w^{*} \in D_{R}(s, t)$ by $\left\{w^{*}\right\}=\{w s, w t\} \cap$ $D_{R}(s, t)$.

Proposition 1.3. (Kazhdan-Lusztig [5]) Let $s$ and $t$ be in $S$ such that st has order 3 , and set $*=\{s, t\}$.

(i) For $w \in D_{L}(s, t)\left(\right.$ resp. $\left.D_{R}(s, t)\right)$ we have ${ }^{*} w \underset{L}{\sim} w\left(\right.$ resp. $\left.w^{*} \underset{R}{\sim} w\right)$.

(ii) For $y, w \in D_{L}(s, t)$ (resp. $\left.D_{R}(s, t)\right)$ with $y \underset{R}{\sim} w($ resp. $y \underset{L}{\sim} w)$ we have ${ }^{*} y \underset{R}{\sim}{ }^{*} w\left(\right.$ resp. $\left.y^{*} \underset{L}{\sim} w^{*}\right)$.

Given $w, u$ in $\tilde{W}$, we write

$$
C_{w}^{\prime} C_{u}^{\prime}=\sum_{v \in \tilde{W}_{a}} h_{w, u, v} C_{v}^{\prime} \quad\left(h_{w, u, v} \in \mathbb{Z}\left[q^{1 / 2}, q^{-1 / 2}\right]\right) .
$$

The $a$-function

$$
a: \tilde{W} \longrightarrow \mathbb{Z}_{\geq 0} \sqcup\{\infty\}
$$

is defined as follows. Let $v \in \tilde{W}$. If for any $i \in \mathbb{Z}_{\geq 0}$ there exist some $w, u \in$ $\tilde{W}$ such that $q^{-i / 2} h_{w, u, v} \notin \mathbb{Z}\left[q^{-1 / 2}\right]$, then we set $a(v)=\infty$. Otherwise we set

$$
a(v)=\min \left\{i \in \mathbb{Z}_{\geq 0} \mid q^{-i / 2} h_{w, u, v} \in \mathbb{Z}\left[q^{-1 / 2}\right] \text { for all } w, u \in \tilde{W}\right\} .
$$


Proposition 1.4. (Lusztig [10]) Assume that $(W, S)$ is crystallographic. Then for any $w, y \in \tilde{W}$ with $y \underset{L R}{\leq} w$ we have $a(y) \geq a(w)$. In particular, the function a is constant on each two-sided cell of $\tilde{W}$.

\section{§2. Affine Hecke algebras}

Let $G$ be a connected reductive algebraic group over $\mathbb{C}$ with simplyconnected derived group. Let $B$ and $T$ be a Borel subgroup and a maximal torus of $G$ respectively such that $B \supset T$. We denote the Lie algebras of $G$, $B, T$ by $\mathfrak{g}, \mathfrak{b}, \mathfrak{t}$ respectively. Let $\Delta \subset \mathfrak{t}^{*}$ be the root system. For $\alpha \in \Delta$ we denote the corresponding root subspace by $\mathfrak{g}_{\alpha}$. We choose a system $\Delta^{+}$ of positive roots as the weights of $\mathfrak{g} / \mathfrak{b}$, and denote the corresponding set of simple roots by $\Pi$. Let $P \subset \mathfrak{t}^{*}$ denote the weight lattice and let $Q$ be its sublattice spanned by $\Delta$. We denote the subset of $P$ consisting of dominant weights by $P^{+}$.

In the rest of this paper we denote by $W$ the Weyl group of $G$. It is a Coxeter group with canonical generator system $S=\left\{s_{\alpha} \mid \alpha \in \Pi\right\}$. Here, the reflection with respect to $\alpha \in \Delta$ is denoted by $s_{\alpha}$.

Let $W_{a}=W Q$ (resp. $\tilde{W}_{a}=W P$ ) denote the semidirect product with respect to the action $W$ on $Q$ (resp. $P$ ). $W_{a}$ and $\tilde{W}_{a}$ are called the affine Weyl group and the extended affine Weyl group respectively. The element of $W_{a}$ (resp. $\tilde{W}_{a}$ ) corresponding to $\lambda \in Q$ (resp. $\lambda \in P$ ) is denoted by $t_{\lambda}$. Let $\Delta_{c}$ denote the set of roots $\beta$ such that the corresponding coroots $\beta^{\vee}$ are the highest coroots of irreducible components of the coroot system, and set

$$
S_{a}=S \sqcup\left\{t_{\beta} s_{\beta} \mid \beta \in \Delta_{c}\right\} .
$$

Then $\left(W_{a}, S_{a}\right)$ is a Coxeter system. Set

$$
\Omega=\left\{\omega \in \tilde{W}_{a} \mid \omega S_{a}=S_{a} \omega\right\}
$$

Then $\tilde{W}_{a}$ is canonically isomorphic to the semidirect product $\Omega W_{a}$ with respect to the conjugation action of $\Omega$ on $W_{a}$. Especially, we have the Hecke algebra $H\left(\tilde{W}_{a}\right)$ of $\tilde{W}_{a}$. We identify the Hecke algebra $H(W)$ of $W$ with a subalgebra of $H\left(\tilde{W}_{a}\right)$ by the canonical embedding $T_{w} \mapsto T_{w}(w \in W)$.

We have the following properties on the $a$-function on $\tilde{W}_{a}$.

Proposition 2.1. (Lusztig [10])

(i) $a(w)=\ell(w)$ if $w$ is a parabolic element of $\tilde{W}_{a}$. 
(ii) $a(w) \leq a\left(w_{S}\right)\left(=\ell\left(w_{S}\right)\right)$ for any $w \in \tilde{W}_{a}$.

Note also that the function $a$ is constant on each two-sided cell of $\tilde{W}_{a}$ by Proposition 1.4. For $w, u, v \in \tilde{W}$ we define $\gamma_{w, u, v} \in \mathbb{Z}$ by

$$
h_{w, u, v}=\gamma_{w, u, v} q^{a(v) / 2}+\text { lower degree terms. }
$$

Now we present a property of $\gamma_{w, u, v}$ related to the star operations.

By a similar argument to that for Theorem 1.4.5 in [22], we can see the following result.

Proposition 2.2. Let $s, t$ be in $S_{a}$ such that st has order 3 . Set $*=\{s, t\}$. Assume $w, v \in D_{L}(s, t)$. Then we have

$$
\gamma_{w, u, v}=\gamma^{*} w, u,{ }^{*} v
$$

For $\lambda \in P$ we define $\theta_{\lambda} \in H\left(\tilde{W}_{a}\right)$ as follows. Take $\lambda_{1}, \lambda_{2} \in P^{+}$such that $\lambda=\lambda_{1}-\lambda_{2}$ and set

$$
\theta_{\lambda}=q^{\left(-\ell\left(t_{\lambda_{1}}\right)+\ell\left(t_{\lambda_{2}}\right)\right) / 2} T_{t_{\lambda_{1}}} T_{t_{\lambda_{2}}}^{-1}
$$

It does not depend on the choice of $\lambda_{1}, \lambda_{2}$. Moreover, we have

$$
\begin{aligned}
& \theta_{0}=1, \\
& \theta_{\lambda} \theta_{\mu}=\theta_{\lambda+\mu} \quad(\lambda, \mu \in P), \\
& T_{s_{\alpha}} \theta_{\lambda}=\theta_{s \lambda} T_{s_{\alpha}}+(q-1) \frac{\theta_{\alpha}\left(\theta_{\lambda}-\theta_{s \lambda}\right)}{\theta_{\alpha}-1} \quad(\lambda \in P, \alpha \in \Pi) .
\end{aligned}
$$

This presentation in terms of the $\mathbb{Z}\left[q^{1 / 2}, q^{-1 / 2}\right]$-basis $\left\{T_{w} \theta_{\lambda} \mid w \in W, \lambda \in P\right\}$ of $H\left(\tilde{W}_{a}\right)$ is due to Bernstein-Zelevinski (see [8]).

\section{$\S 3$. Affine Hecke algebras and equivariant $K$-groups}

For an algebraic variety $Y$ over $\mathbb{C}$ we denote its structure sheaf by $\mathcal{O}_{Y}$. If $Y$ is smooth, then its canonical sheaf is denoted by $\Omega_{Y}$.

We denote the flag variety $G / B$ of $G$ by $\mathcal{B}$. As a set $\mathcal{B}$ is identified with the set of Borel subalgebras of $\mathfrak{g}$ by the correspondence $g B \mapsto \operatorname{Ad}(g)(\mathfrak{b})$ $(g \in G)$. For $x \in \mathcal{B}$ we denote by $\mathfrak{b}_{x}$ the corresponding Borel subalgebra of $\mathfrak{g}$, and set $\mathfrak{n}_{x}=\left[\mathfrak{b}_{x}, \mathfrak{b}_{x}\right]$. For $w \in W$ set

$$
Y_{w}=G(B, w B) \subset \mathcal{B} \times \mathcal{B}
$$


Then we have $\mathcal{B} \times \mathcal{B}=\bigsqcup_{w \in W} Y_{w}$, and $\bar{Y}_{w}=\bigsqcup_{y \leq w} Y_{y}$. We denote by

$$
i_{w}: \bar{Y}_{w} \longrightarrow \mathcal{B} \times \mathcal{B}
$$

the embedding.

Set

$$
\begin{aligned}
& \Lambda=\left\{(a, x) \in \mathfrak{g} \times \mathcal{B} \mid a \in \mathfrak{n}_{x}\right\} \\
& Z=\left\{(a, x, y) \in \mathfrak{g} \times \mathcal{B} \times \mathcal{B} \mid a \in \mathfrak{n}_{x} \cap \mathfrak{n}_{y}\right\}
\end{aligned}
$$

Let $\pi: \Lambda \rightarrow \mathcal{B}$ be the projection. The algebraic group $G \times \mathbb{C}^{*}$ acts on the variety $\Lambda$ by

$$
(g, z):(a, x) \longmapsto\left(z^{-2} \operatorname{Ad}(g)(a), g x\right) \in \Lambda .
$$

We sometimes identify $Z$ with a $G \times \mathbb{C}^{*}$-stable closed subvariety of $\Lambda \times \Lambda$ by the embedding

$$
Z \longrightarrow \Lambda \times \Lambda \quad((a, x, y) \longmapsto((a, x),(a, y)))
$$

In particular, $Z$ is a $G \times \mathbb{C}^{*}$-variety. For $w \in W$ set

$$
Z_{w}=\left\{(a, x, y) \in Z \mid(x, y) \in Y_{w}\right\}
$$

We denote by

$$
r_{w}: \bar{Z}_{w} \longrightarrow Z, \quad \pi_{w}: \bar{Z}_{w} \longrightarrow \bar{Y}_{w}
$$

the embedding and the projection respectively.

Let us consider the equivariant $K$-group $K^{G \times \mathbb{C}^{*}}(Z)=K^{G \times \mathbb{C}^{*}}(\Lambda \times \Lambda ; Z)$ (see Section A for the equivariant $K$-groups and notation concerning them). It is a module over the representation ring $R^{G \times \mathbb{C}^{*}}=R^{G} \otimes_{\mathbb{Z}} R^{\mathbb{C}^{*}}$ of $G \times \mathbb{C}^{*}$. We will identify $R^{\mathbb{C}^{*}}$ with $\mathbb{Z}\left[q^{1 / 2}, q^{-1 / 2}\right]$ by associating the $\mathbb{C}^{*}$-module given by $z \mapsto z^{n}$ to $q^{n / 2}$. In particular, $K^{G \times \mathbb{C}^{*}}(Z)$ is a $\mathbb{Z}\left[q^{1 / 2}, q^{-1 / 2}\right]$-module.

For $(i, j)=(1,2),(2,3),(1,3)$ we denote by $p_{i j}: \Lambda \times \Lambda \times \Lambda \rightarrow \Lambda \times \Lambda$ the projections onto $(i, j)$-factors. Note that $p_{13}\left(p_{12}^{-1} Z \cap p_{23}^{-1} Z\right) \subset Z$. Since the morphism $p_{12}^{-1} Z \cap p_{23}^{-1} Z \rightarrow Z$ induced by $p_{13}$ is proper, we can define an $R^{G \times \mathbb{C}^{*}}$-bilinear map

$$
\begin{aligned}
\star & : K^{G \times \mathbb{C}^{*}}(Z) \times K^{G \times \mathbb{C}^{*}}(Z) \longrightarrow K^{G \times \mathbb{C}^{*}}(Z) \\
& \left((m, n) \longmapsto m \star n=p_{13 *}\left(p_{12}^{*} m \otimes_{\mathcal{O}_{\Lambda \times \Lambda \times \Lambda}} p_{23}^{*} n\right)\right) .
\end{aligned}
$$


Then it is easily seen that the convolution product $\star$ endows with $K^{G \times \mathbb{C}^{*}}(Z)$ a structure of associative algebra over $R^{G \times \mathbb{C}^{*}}$ with the identity element $\left[r_{1 *} \mathcal{O}_{Z_{1}}\right]$. For $\lambda \in P$ we denote by $\mathcal{O}_{\mathcal{B}}(\lambda)$ the $G$-equivariant invertible $\mathcal{O}_{\mathcal{B}}$-module whose fiber at $B$ is the $B$-module corresponding to $\lambda$.

Theorem 3.1. (Ginzburg [3], Kazhdan-Lusztig [6]) There exists an isomorphism

$$
\Phi: H\left(\tilde{W}_{a}\right) \longrightarrow K^{G \times \mathbb{C}^{*}}(Z)
$$

of $\mathbb{Z}\left[q^{1 / 2}, q^{-1 / 2}\right]$-algebras satisfying

$$
\begin{aligned}
\Phi\left(\theta_{\lambda}\right) & =\left[r_{1 *} \pi_{1}^{*} \mathcal{O}_{\mathcal{B}}(-\lambda)\right] \quad(\lambda \in P), \\
\Phi\left(T_{s}+1\right) & =-\left[r_{s *} \pi_{s}^{*}\left(\Omega_{\bar{Y}_{s}} \otimes i_{s}^{*}\left(\mathcal{O}_{\mathcal{B}} \otimes \Omega_{\mathcal{B}}^{\otimes-1}\right)\right)\right] \quad(s \in S) .
\end{aligned}
$$

Here, we have identified $\bar{Y}_{1}\left(=Y_{1}\right)$ with $\mathcal{B}$.

Remark 3.2. Note that $\Phi\left(T_{s}+1\right)$ is not symmetric with respect to the the symmetry of $(\Lambda \times \Lambda, Z)$ given by $\Lambda \times \Lambda \ni(x, y) \mapsto(y, x) \in \Lambda \times \Lambda$. This can be resolved if we use the twisted product

$$
\begin{aligned}
& K^{G \times \mathbb{C}^{*}}(Z) \times K^{G \times \mathbb{C}^{*}}(Z) \longrightarrow K^{G \times \mathbb{C}^{*}}(Z) \\
&((m, n)\left.\longmapsto p_{13 *}\left(p_{12}^{*} m \otimes_{\mathcal{O}_{\Lambda \times \Lambda \times \Lambda}} p_{23}^{*} n \otimes_{\mathcal{O}_{\Lambda \times \Lambda \times \Lambda}} p_{2}^{*} \pi^{*} \Omega_{\mathcal{B}}\right)\right)
\end{aligned}
$$

as in [18], where $p_{2}: \Lambda \times \Lambda \times \Lambda \rightarrow \Lambda$ is the projection onto the second factor. There is another way to recover the symmetry by modifying the definition of $\Phi$ without changing the product (see Lusztig [13]).

Let $\mathcal{N}$ denote the closed subvariety of $\mathfrak{g}$ consisting of nilpotent elements. For a locally closed $G$-stable subvariety $V$ of $\mathcal{N}$ we set

$$
Z_{V}=\{(a, x, y) \in Z \mid a \in V\}
$$

Proposition 3.3. (Ginzburg [3], Kazhdan-Lusztig [6]) Let $V$ be a locally closed $G$-stable subvariety of $\mathcal{N}$. Then we have an exact sequence

$$
0 \longrightarrow K^{G \times \mathbb{C}^{*}}\left(Z_{\bar{V} \backslash V}\right) \longrightarrow K^{G \times \mathbb{C}^{*}}\left(Z_{\bar{V}}\right) \longrightarrow K^{G \times \mathbb{C}^{*}}\left(Z_{V}\right) \longrightarrow 0 .
$$

Here $K^{G \times \mathbb{C}^{*}}\left(Z_{\bar{V} \backslash V}\right) \rightarrow K^{G \times \mathbb{C}^{*}}\left(Z_{\bar{V}}\right)$ is given by the direct image with respect to the inclusion $Z_{\bar{V} \backslash V} \rightarrow Z_{\bar{V}}$, and $K^{G \times \mathbb{C}^{*}}\left(Z_{\bar{V}}\right) \rightarrow K^{G \times \mathbb{C}^{*}}\left(Z_{V}\right)$ is given by the inverse image with respect to the inclusion $Z_{V} \rightarrow Z_{\bar{V}}$. 
In particular, if $V$ is closed, then the homomorphism $K^{G \times \mathbb{C}^{*}}\left(Z_{V}\right) \rightarrow$ $K^{G \times \mathbb{C}^{*}}(Z)$ given by the direct image with respect to the closed embedding $Z_{V} \rightarrow Z$ is injective. By this we will identify $K^{G \times \mathbb{C}^{*}}\left(Z_{V}\right)$ for a closed $G$-stable subvariety $V$ of $\mathcal{N}$ with a two-sided ideal of $K^{G \times \mathbb{C}^{*}}(Z)$.

The following remarkable fact conjectured in Lusztig [7] was proved by Lusztig himself [12] using the theory of character sheaves among other things.

THEOREM 3.4. There exists a natural one-to-one correspondence between the set of two-sided cells of $\tilde{W}_{a}$ and that of nilpotent orbits of $\mathfrak{g}$.

For a nilpotent orbit $O$ we denote by $\mathcal{C}_{O}$ the corresponding two-sided cell.

In view of Theorem 3.1, it is natural to expect the following (see [4], [19], [14]).

Conjecture 3.5. Let $O$ be a nilpotent orbit. Then we have

$$
\Phi\left(H\left(\tilde{W}_{a}\right)_{L R}^{\leq \mathcal{C}_{O}}\right)=K^{G \times \mathbb{C}^{*}}\left(Z_{\bar{O}}\right) .
$$

Remark 3.6. This conjecture is known to be true when $O=\{0\}$ (see [21]). In [1] Bezrukavnikov established a closely related result, which involves affine flag manifolds, derived categories and the Springer resolution (see Theorem 4(a) there).

Let $w \in W$. In [18] a $G \times \mathbb{C}^{*}$-equivariant coherent sheaf $M_{w}$ on $\Lambda \times \Lambda$ such that $\operatorname{Supp}\left(M_{w}\right) \subset Z$ and $\Phi\left(C_{w}\right)=(-1)^{\ell(w)}\left[M_{w}\right]$ is associated using the theory of Hodge modules. This together with a deep result related to the associated varieties of primitive ideals of the enveloping algebra $U(\mathfrak{g})$ implies

$$
\Phi\left(C_{w}\right) \in K^{G \times \mathbb{C}^{*}}\left(Z_{\bar{O}}\right) \backslash K^{G \times \mathbb{C}^{*}}\left(Z_{\bar{O} \backslash O}\right),
$$

where $O$ is the nilpotent orbit satisfying $w \in \mathcal{C}_{O}$. In Section 4 we will need the following weaker result which is much easier.

Proposition 3.7. Let $\Pi_{1}$ be a subset of $\Pi$. Set $w=w_{T} \in W$ with $T=\left\{s_{\alpha} \mid \alpha \in \Pi_{1}\right\} \subset S$. Let $O$ be the nilpotent orbit satisfying

$$
\bar{O}=\operatorname{Ad}(G)\left(\sum_{\alpha \in \Delta^{+} \backslash \Delta_{1}} \mathfrak{g}_{\alpha}\right),
$$


where $\Delta_{1}=\Delta \cap\left(\sum_{\alpha \in \Pi_{1}} \mathbb{Z} \alpha\right)$. Then we have

$$
\Phi\left(C_{w}\right) \in K^{G \times \mathbb{C}^{*}}\left(Z_{\bar{O}}\right) .
$$

Proof. Note that $\bar{Y}_{w}$ is smooth. Hence by [18] we have $\Phi\left(C_{w}\right)=$ $(-1)^{\ell(w)}\left[M_{w}\right]$ with

$$
M_{w}=\operatorname{gr}\left(\mathbb{Q} \bar{Y}_{w}\left[\operatorname{dim} \bar{Y}_{w}\right]\right) \otimes \mathcal{O}_{\Lambda \times \Lambda}(\pi \times \pi)^{*}\left(\mathcal{O}_{\mathcal{B}} \otimes \Omega_{\mathcal{B}}^{\otimes-1}\right),
$$

where $\mathbb{Q} \frac{H}{Y_{w}}\left[\operatorname{dim} \bar{Y}_{w}\right]$ denotes the canonical irreducible $G$-equivariant Hodge module whose underlying perverse sheaf is $\mathbb{Q}_{\bar{Y}_{w}}\left[\operatorname{dim} \bar{Y}_{w}\right]$. By

$$
\operatorname{gr}\left(\mathbb{Q} \frac{H}{Y_{w}}\left[\operatorname{dim} \bar{Y}_{w}\right]\right)=r_{w *} \pi_{w}^{*}\left(\Omega_{\bar{Y}_{w}}\right)
$$

we obtain

$$
M_{w}=r_{w *} \pi_{w}^{*}\left(\Omega_{\bar{Y}_{w}}\right) \otimes \mathcal{O}_{\Lambda \times \Lambda}(\pi \times \pi)^{*}\left(\mathcal{O}_{\mathcal{B}} \otimes \Omega_{\mathcal{B}}^{\otimes-1}\right)
$$

It follows that $\operatorname{Supp}\left(M_{w}\right)=\bar{Z}_{w} \subset Z_{\bar{O}}$.

Remark 3.8. We can prove (3.1) directly without appealing to the theory of Hodge modules. Details are omitted.

\section{§4. The case $G=G L_{n}(\mathbb{C})$}

The main result of this paper is the following.

TheOREM 4.1. Conjecture 3.5 holds for $G=G L_{n}(\mathbb{C})$.

In the rest of this section we assume that $G=G L_{n}(\mathbb{C})$. In this case the extended affine Weyl group $\tilde{W}_{a}$ is identified with the group of all permutations $\sigma$ of $\mathbb{Z}$ satisfying $\sigma(i+n)=\sigma(i)+n(i \in \mathbb{Z})$ and $\sum_{i=1}^{n}(\sigma(i)-i) \in n \mathbb{Z}$. Define $\omega, s_{k} \in \tilde{W}_{a}(0 \leq k \leq n-1)$ by

$$
\begin{aligned}
& \omega(i)=i+1 \quad(i \in \mathbb{Z}), \\
& s_{k}(i)= \begin{cases}i+1 & (i \in n \mathbb{Z}+k), \\
i-1 & (i \in n \mathbb{Z}+k+1), \\
i & \text { (otherwise) }\end{cases}
\end{aligned}
$$

Then we have

$$
S=\left\{s_{i} \mid 1 \leq i \leq n-1\right\}, \quad S_{a}=S \sqcup\left\{s_{0}\right\}, \quad \Omega=\langle\omega\rangle,
$$


and $W$ is identified with the symmetric group $\mathfrak{S}_{n}$.

Let $\mathcal{P}(n)$ denote the set of partitions of $n$, that is,

$$
\mathcal{P}(n)=\left\{\rho=\left(\rho_{1}, \rho_{2}, \ldots, \rho_{n}\right) \in \mathbb{Z}_{\geq 0}^{n} \mid \rho_{i} \geq \rho_{i+1}, \sum_{i=1}^{n} \rho_{i}=n\right\} .
$$

For $\rho \in \mathcal{P}(n)$ we set

$$
N_{j}(\rho)=\sharp\left\{i \mid \rho_{i}=j\right\} .
$$

We denote by $\rho \mapsto \rho^{*}$ the duality operation on $\mathcal{P}(n)$ induced by the transpose of the corresponding Young diagram, that is, $\rho_{i}^{*}=\sum_{k=i}^{n} N_{k}(\rho)$.

The set of nilpotent orbits in $\mathfrak{g}=\mathfrak{g l}_{n}(\mathbb{C})$ is parametrized by $\mathcal{P}(n)$. The nilpotent orbit $O_{\rho}$ corresponding to $\rho \in \mathcal{P}(n)$ is the one containing the Jordan normal form with exactly $N_{i}\left(\rho^{*}\right)$ Jordan blocks of size $i$ (with eigenvalue 0) for each $i$. In particular, $O_{(n, 0, \ldots, 0)}=\{0\}$ and $O_{(1, \ldots, 1)}$ is the regular nilpotent orbit.

By Theorem 3.4 the set of two-sided cells of $\tilde{W}_{a}$ is also parametrized by $\mathcal{P}(n)$ (in our case $G=G L_{n}(\mathbb{C})$ this is due to Lusztig [9] and Shi [15]). We denote by $\mathcal{C}_{\rho}$ the two-sided cell of $\tilde{W}_{a}$ corresponding to $O_{\rho}$.

Let $T$ be a proper subset of $S_{a}$ such that $\langle T\rangle$ is of type $A_{k_{1}} \times \cdots \times A_{k_{r}}$. Then the corresponding parabolic element $w_{T}$ belongs to $\mathcal{C}_{\rho}$ if and only if

$$
\sharp\left\{j \mid k_{j}+1=i\right\}=N_{i}(\rho)
$$

for any $i$.

For $\rho \in \mathcal{P}(n)$ set $\mathcal{C}_{\rho}^{W}=W \cap \mathcal{C}_{\rho}$. It is known that $\mathcal{C}_{\rho}^{W}$ is a two-sided cell of $W$. In particular, the set of two-sided cells of $W$ is also parametrized by $\mathcal{P}(n)$ (see Kazhdan-Lusztig [5]).

Proposition 4.2. (Shi [16]) The following conditions on $\rho, \xi \in \mathcal{P}(n)$ are equivalent.

(a) $\mathcal{C}_{\xi} \underset{L R}{\leq} \mathcal{C}_{\rho}$

(b) $\mathcal{C}_{\xi}^{W} \underset{L R}{\leq} \mathcal{C}_{\rho}^{W}$

(c) $O_{\xi} \subset \bar{O}_{\rho}$.

Hence we have $H(W)_{L_{L R}}^{\leq \mathcal{C}_{\rho}^{W}}=H\left(\tilde{W}_{a}\right)_{L R}^{\leq \mathcal{C}_{\rho}} \cap H(W)$, and $H(W)_{\mathcal{C}_{\rho}^{W}}$ is identified with an $(H(W), H(W))$-submodule of $H\left(\tilde{W}_{a}\right)_{\mathcal{C}_{\rho}}$.

The following is crucial for the proof of Theorem 4.1 . 
TheOREM 4.3. Let $v$ be a parabolic element in $\mathcal{C}_{\rho}$. Then the $H\left(\tilde{W}_{a}\right)$ bimodule $H\left(\tilde{W}_{a}\right)_{\mathcal{C}_{\rho}}$ is generated by the image of $C_{v}$.

We first show the following corresponding statement for $H(W)$.

Proposition 4.4. Let $v$ be a parabolic element in $\mathcal{C}_{\rho}^{W}$. Then the $H(W)$-bimodule $H(W)_{\mathcal{C}_{\rho}^{W}}$ is generated by the image of $C_{v}$.

Proof. Let $u \in \mathcal{C}_{\rho}^{W}$. Let $\mathcal{L}$ be the left cell of $W$ containing $u$ and $\mathcal{R}$ the right cell of $W$ containing $u$. Then $\mathcal{L}$ contains a unique element $y$ such that $y \underset{R}{\sim} v$, and $\mathcal{R}$ contains a unique element $x$ such that $x \underset{L}{\sim} v$ (see the proof of Theorem 1.4 in $[5, \S 5])$. By Lemma 1.2 we have $C_{x}^{\prime}=h C_{v}^{\prime}$ and $C_{y}^{\prime}=C_{v}^{\prime} h^{\prime}$ for some $h, h^{\prime}$ in $H(W)$.

Let $\pi: H(W)_{\leq \mathcal{C}_{\rho}^{W}} \rightarrow H(W)_{\mathcal{C}_{\rho}^{W}}$ be the canonical projection and let $V_{1}$ and $V_{2}$ be the left $H(W)$-submodules of $H(W)_{\mathcal{C}_{\rho}^{W}}$ generated by $\pi\left(C_{v}^{\prime}\right)$ and $\pi\left(C_{y}^{\prime}\right)$ respectively. Then $H(W)_{\mathcal{C}_{\rho}^{W}} \ni k \mapsto k h^{\prime} \in H(W)_{\mathcal{C}_{\rho}^{W}}$ is a homomorphism of left $H(W)$-modules satisfying $\pi\left(C_{v}^{\prime}\right) \mapsto \pi\left(C_{y}^{\prime}\right)$. Hence we obtain a homomorphism $f: V_{1} \rightarrow V_{2}$ given by $f(k)=k h^{\prime}$.

On the other hand by the proof of Theorem 1.4 in $[5, \S 5]$ there exists an isomorphism $g: V_{1} \rightarrow V_{2}$ of left $H(W)$-modules such that $g\left(\pi\left(C_{v}^{\prime}\right)\right)=\pi\left(C_{y}^{\prime}\right)$ and $g\left(\pi\left(C_{x}^{\prime}\right)\right)=\pi\left(C_{u}^{\prime}\right)$. By $f\left(\pi\left(C_{v}^{\prime}\right)\right)=g\left(\pi\left(C_{v}^{\prime}\right)\right)$ we have $f=g$. Hence

$$
\pi\left(C_{u}^{\prime}\right)=g\left(\pi\left(C_{x}^{\prime}\right)\right)=f\left(\pi\left(C_{x}^{\prime}\right)\right)=h f\left(\pi\left(C_{v}^{\prime}\right)\right)=h \pi\left(C_{v}^{\prime}\right) h^{\prime} .
$$

The proof is complete.

Now we give a proof of Theorem 4.3. Let $\pi: H\left(\tilde{W}_{a}\right)_{\leq \mathcal{C}_{\rho}} \rightarrow H\left(\tilde{W}_{a}\right)_{\mathcal{C}_{\rho}}$ be the canonical homomorphism. According to [15, Lemma 18.3.2] one has a parabolic element $w \in \mathcal{C}_{\rho}^{W}$ such that for any $u \in \mathcal{C}_{\rho}$ there exists a sequence of left star operations $\phi_{1}, \phi_{2}, \ldots, \phi_{r}$ and an integer $m$ satisfying

$$
w \underset{R}{\sim} \omega^{m} \phi_{r} \phi_{r-1} \cdots \phi_{1}(u) .
$$

We first show the statement for this special parabolic element $w$.

Let $u \in \mathcal{C}_{\rho}$. Take left star operations $\phi_{1}, \phi_{2}, \ldots, \phi_{r}$ and an integer $m$ satisfying (4.1), and set $y=\omega^{m} \phi_{r} \phi_{r-1} \cdots \phi_{1}(u), x=\phi_{1} \phi_{2} \cdots \phi_{r} \omega^{-m}(w)$. Note that $x$ is well-defined and $x \underset{L}{\sim} w$ by definition and Proposition 1.3. Since $w$ is a parabolic element, there exist $h, h^{\prime} \in H\left(\tilde{W}_{a}\right)$ such that $C_{x}^{\prime}=$ 
$h C_{w}^{\prime}$ and $C_{y}^{\prime}=C_{w}^{\prime} h^{\prime}$ by Lemma 1.2. Note that $C_{w}^{\prime} C_{w}^{\prime}=\eta C_{w}^{\prime}$ where $\eta \in$ $\mathbb{Z}\left[q^{1 / 2}, q^{-1 / 2}\right]$ satisfies $\bar{\eta}=\eta$ and $\eta=q^{\ell(w) / 2}+$ (lower degree terms). Hence

$$
\eta h \pi\left(C_{w}^{\prime}\right) h^{\prime}=\pi\left(C_{x}^{\prime} C_{y}^{\prime}\right)=\sum_{z \in \mathcal{C}_{\rho}} h_{x, y, z} \pi\left(C_{z}^{\prime}\right)
$$

where $h_{x, y, z} \in \mathbb{Z}\left[q^{1 / 2}, q^{-1 / 2}\right]$ satisfies $\bar{h}_{x, y, z}=h_{x, y, z}$ and $h_{x, y, z}=\gamma_{x, y, z} q^{a(z) / 2}$ + (lower degree terms). For any $z \in \mathcal{C}_{\rho}$ we have $a(z)=a(w)=\ell(w)$, and hence we obtain

$$
h \pi\left(C_{w}^{\prime}\right) h^{\prime}=\sum_{z \in \mathcal{C}_{\rho}} \gamma_{x, y, z} \pi\left(C_{z}^{\prime}\right)
$$

Note that $\gamma_{\omega^{m}} w_{1}, w_{2}, w_{3}=\gamma_{w_{1}, w_{2}, \omega^{-m} w_{3}}$ for any $w_{1}, w_{2}, w_{3}$ in $\tilde{W}_{a}$. Hence we have $\gamma_{x, y, z}=\gamma_{w, y, \omega^{m} \phi_{r} \cdots \phi_{1}(z)}$ by Proposition 2.2. Since $w$ is a distinguished involution, we have $\gamma_{x, y, z} \neq 0$ if and only if $\omega^{m} \phi_{r} \phi_{r-1} \cdots \phi_{1}(z)=y$ and in this case $\gamma_{x, y, z}=1$ (see Lusztig [11]). Thus $\gamma_{x, y, z} \neq 0$ if and only $z=u$ and in this case $\gamma_{x, y, u}=1$. Therefore we have $h \pi\left(C_{w}^{\prime}\right) h^{\prime}=\pi\left(C_{u}^{\prime}\right)$.

Now let $v$ be any parabolic element in $\mathcal{C}_{\rho}$. Then there exists an integer $k$ such that $\omega^{k} v \omega^{-k}$ is in $W$. By Proposition 4.4 we have $H(W) \pi\left(C_{\omega^{k} v \omega^{-k}}^{\prime}\right)$ $H(W)=H(W) \pi\left(C_{w}^{\prime}\right) H(W)$ and hence

$$
H\left(\tilde{W}_{a}\right) \pi\left(C_{v}^{\prime}\right) H\left(\tilde{W}_{a}\right)=H\left(\tilde{W}_{a}\right) \pi\left(C_{w}^{\prime}\right) H\left(\tilde{W}_{a}\right)=H\left(\tilde{W}_{a}\right)_{\mathcal{C}_{\rho}}
$$

The proof of Theorem 4.3 is complete.

Remark 4.5. (a) The assertion for $W_{a}$ similar to that in Theorem 4.3 does not hold in general.

(b) Let $v$ be as in Theorem 4.3. It is not difficult to prove that for any $w \underset{L R}{\leq} v$, there exists a polynomial $f_{w}$ in $q^{1 / 2}+q^{-1 / 2}$ such that $f_{w} C_{w}$ is in the two-sided ideal of $H\left(\tilde{W}_{a}\right)$ generated by $C_{v}$. However, in general it is not true that $C_{w}$ is in the two-sided ideal of $H\left(\tilde{W}_{a}\right)$ generated by $C_{v}$. Example: $n=4$ and let $\mathcal{C}_{\rho}$ be the two-sided cell containing $v=$ $s_{1} s_{3}$. Then $\left(q^{1 / 2}+q^{-1 / 2}\right) C_{s_{1} s_{2} s_{1}}$ is in $H\left(\tilde{W}_{a}\right) C_{v} H\left(\tilde{W}_{a}\right)$, but $C_{s_{1} s_{2} s_{1}}$ is not in $H\left(\tilde{W}_{a}\right) C_{v} H\left(\tilde{W}_{a}\right)$.

Let $\mathbb{F}$ be an algebraic closure of $\mathbb{C}\left(q^{1 / 2}\right)$, and set $H^{\mathbb{F}}=\mathbb{F} \otimes H\left(\tilde{W}_{a}\right)$, $G_{\mathbb{F}}=G L_{n}(\mathbb{F}), \mathfrak{g}_{\mathbb{F}}=\mathfrak{g l}_{n}(\mathbb{F})$. Then $H^{\mathbb{F}}$ is an $\mathbb{F}$-algebra and $G_{\mathbb{F}}$ is an algebraic group over $\mathbb{F}$ with Lie algebra $\mathfrak{g}_{\mathbb{F}}$.

Let $\mathcal{Q}$ denote the $G_{\mathbb{F}}$-conjugacy classes of the pairs $(s, e) \in G_{\mathbb{F}} \times \mathfrak{g}_{\mathbb{F}}$ where $s$ is semisimple, $e$ is nilpotent, and $\operatorname{Ad}(s)(e)=q e$. For such a pair 
$(s, e)$ Kazhdan-Lusztig [6] and Ginzburg [3] constructed a finite-dimensional $H^{\mathbb{F}}$-module $M_{(s, e)}$. Moreover, we have a unique irreducible quotient $L_{(s, e)}$ of $M_{(s, e)}$, and the set of irreducible $H^{\mathbb{F}}$-modules is parametrized by $\mathcal{Q}$ via $(s, e) \mapsto L_{(s, e)}$ (note that $\mathbb{F}$ is isomorphic to $\mathbb{C}$ as an abstract field). In particular, we can associate to each irreducible $H^{\mathbb{F}}$-module $L$ a nilpotent orbit $O(L)$ in $\mathfrak{g}$ by $\operatorname{Ad}\left(G_{\mathbb{F}}\right)\left(O\left(L_{(s, e)}\right)\right) \ni e$ (note that the set of $G_{\mathbb{F}}$-conjugacy classes of nilpotent elements in $\mathfrak{g}_{\mathbb{F}}$ is in one-to-one correspondence with that of $G$-conjugacy classes of nilpotent elements in $\mathfrak{g}$ ).

We need the following deep result of Lusztig [12].

Proposition 4.6. For any irreducible subquotient $L$ of the (left) $H^{\mathbb{F}_{-}}$ module $\mathbb{F} \otimes_{\mathbb{Z}\left[q^{1 / 2}, q^{-1 / 2}\right]} H\left(\tilde{W}_{a}\right)_{\mathcal{C}_{\rho}}$ we have $\overline{O(L)} \supset O_{\rho}$.

Proposition 4.7. Let $O$ be a nilpotent orbit. Then for any irreducible quotient $L$ of the (left) $H^{\mathbb{F}}$-module $\mathbb{F} \otimes_{\mathbb{Z}\left[q^{1 / 2}, q^{-1 / 2}\right]} K^{G \times \mathbb{C}^{*}}\left(Z_{O}\right)$ we have $O(L)=O$.

Proof. By [6, Corollary 5.9] we see that $L$ is a quotient of $M_{(s, e)}$ for $(s, e) \in \mathcal{Q}$ with $e \in O$. Since $L_{(s, e)}$ is the unique irreducible quotient of $M_{(s, e)}$, we have $L=L_{(s, e)}$ and hence $O(L)=O$.

Now we are ready to give a proof of Theorem 4.1. We show

$$
\Phi\left(H\left(\tilde{W}_{a}\right)_{L R}^{\leq \mathcal{C}_{\xi}}\right)=K^{G \times \mathbb{C}^{*}}\left(Z_{\bar{O}_{\xi}}\right)
$$

for any $\xi \in \mathcal{P}(n)$ by induction on $\operatorname{dim} O_{\xi}$. Let $\rho \in \mathcal{P}(n)$ and assume that (4.2) is true for any $\xi \in \mathcal{P}(n)$ with $\operatorname{dim} O_{\xi}<\operatorname{dim} O_{\rho}$.

For any $\tau \in \mathcal{P}(n)$ with $\mathcal{C}_{\tau} \underset{L R}{\leq} \mathcal{C}_{\rho}$ any parabolic element $v \in \mathcal{C}_{\tau}^{W}$ satisfies $\Phi\left(C_{v}\right) \in K^{G \times \mathbb{C}^{*}}\left(Z_{\bar{O}_{\rho}}\right)$ by Proposition 3.7. Hence we see by Theorem 4.3 that $\Phi\left(H\left(\tilde{W}_{a}\right)_{L R}^{\leq \mathcal{C}_{\rho}}\right) \subset K^{G \times \mathbb{C}^{*}}\left(Z_{\bar{O}_{\rho}}\right)$. Moreover, the hypothesis of induction together with Proposition 4.2 implies $\Phi\left(H\left(\tilde{W}_{a}\right)_{L R}^{<\mathcal{C}_{\rho}}\right)=K^{G \times \mathbb{C}^{*}}\left(Z_{\bar{O}_{\rho} \backslash O_{\rho}}\right)$. Hence it is sufficient to show that the induced injection $\bar{\Phi}: H\left(\tilde{W}_{a}\right)_{\mathcal{C}_{\rho}} \rightarrow$ $K^{G \times \mathbb{C}^{*}}\left(Z_{O_{\rho}}\right)$ is surjective. Assume that $\operatorname{Coker}(\bar{\Phi}) \neq 0$. Since $H\left(\tilde{W}_{a}\right)_{L R}^{\leq \mathcal{C}_{\rho}}$ is a direct summand of the $\mathbb{Z}\left[q^{1 / 2}, q^{-1 / 2}\right]$-module $H\left(\tilde{W}_{a}\right)$ and $\Phi\left(H\left(\tilde{W}_{a}\right)\right)=$ $K^{G \times C^{*}}(Z)$, we see that the cokernel of the injective homomorphism

$$
\bar{\Phi}^{\mathbb{F}}: \mathbb{F} \otimes_{\mathbb{Z}\left[q^{1 / 2}, q^{-1 / 2}\right]} H\left(\tilde{W}_{a}\right)_{\mathcal{C}_{\rho}} \longrightarrow \mathbb{F} \otimes_{\mathbb{Z}\left[q^{1 / 2}, q^{-1 / 2}\right]} K^{G \times \mathbb{C}^{*}}\left(Z_{O_{\rho}}\right)
$$


is also non-trivial. Take an irreducible quotient $L$ of the $H^{\mathbb{F}}$-module $\operatorname{Coker}\left(\bar{\Phi}^{\mathbb{F}}\right)$. Since $L$ is an irreducible quotient of $\mathbb{F} \otimes_{\mathbb{Z}\left[q^{1 / 2}, q^{-1 / 2}\right]} K^{G \times \mathbb{C}^{*}}\left(Z_{O_{\rho}}\right)$, we have $O(L)=O_{\rho}$ by Proposition 4.7. On the other hand since $L$ is an irreducible subquotient of the $H^{\mathbb{F}}$-module

$$
\mathbb{F} \otimes_{\mathbb{Z}\left[q^{1 / 2}, q^{-1 / 2}\right]} H\left(\tilde{W}_{a}\right) / \mathbb{F} \otimes_{\mathbb{Z}\left[q^{1 / 2}, q^{-1 / 2}\right]} H\left(\tilde{W}_{a}\right)_{L R}^{\leq \mathcal{C}_{\rho}},
$$

there exists a nilpotent orbit $O$ such that $O \not \subset \overline{O_{\rho}}$ and $O \subset \overline{O(L)}$ by Proposition 4.6. This is a contradiction. Hence $\bar{\Phi}$ is surjective. The proof of Theorem 4.1 is complete.

\section{Appendix A. Equivariant $K$-theory}

In this section we recall basic notions concerning equivariant $K$-groups (see Thomason [20]). All algebraic varieties are assumed to be quasiprojective over $\mathbb{C}$ and all algebraic groups are assumed to be affine over $\mathbb{C}$. The structure sheaf of an algebraic variety $X$ is denoted by $\mathcal{O}_{X}$. When we consider an action of an algebraic group $A$ on an algebraic variety $X$, we always assume the existence of a closed $A$-equivariant embedding $X \rightarrow X^{\prime}$ where $X^{\prime}$ is a smooth variety with an action of $A$. In this case we say that $X$ is an $A$-variety.

Let $A$ be an algebraic group. For a pair $(Y, X)$ such that $Y$ is an $A$ variety and $X$ is its $A$-stable closed subvariety, we denote by $\operatorname{Coh}^{A}(Y ; X)$ the abelian category of $A$-equivariant coherent sheaves on $Y$ whose supports are contained in $X$. Its Grothendieck group $K^{A}(Y ; X)$ is called the equivariant $K$-group. Note that the direct image functor $i_{*}: \operatorname{Coh}^{A}(X ; X) \rightarrow$ $\operatorname{Coh}^{A}(Y ; X)$ with respect to the embedding $i: X \rightarrow Y$ induces an isomorphism $K^{A}(X ; X) \cong K^{A}(Y ; X)$. It means that $K^{A}(Y ; X)$ depends only on the $A$-variety $X$, and hence we sometimes denote it by $K^{A}(X)$. However, we will need to specify the ambient space $Y$ in defining some operations on equivariant $K$-groups. Note that $K^{A}(X)$ is a module over the representation ring

$$
R^{A}=K^{A}(\mathrm{pt})
$$

of $A$. Here pt denotes the variety consisting of a single point.

Assume that we are given an $A$-equivariant morphism $f: Y \rightarrow Y^{\prime}$ of $A$ varieties and $A$-stable closed subvarieties $X$ and $X^{\prime}$ of $Y$ and $Y^{\prime}$ respectively 
such that $f(X) \subset X^{\prime}$ and the restriction $X \rightarrow X^{\prime}$ of $f$ is a proper morphism. Then the derived functors

$$
R^{n} f_{*}: \operatorname{Coh}^{A}(Y ; X) \longrightarrow \operatorname{Coh}^{A}\left(Y^{\prime} ; X^{\prime}\right) \quad(n \in \mathbb{Z})
$$

of the direct image functor $f_{*}$ induce a homomorphism

$$
\text { (A.2) } \quad f_{*}: K^{A}(Y ; X) \longrightarrow K^{A}\left(Y^{\prime} ; X^{\prime}\right) \quad\left([M] \longmapsto \sum_{n}(-1)^{n}\left[R^{n} f_{*}(M)\right]\right)
$$

of $R^{A}$-modules. We note that (A.2) does not depend on the choice of the ambient spaces $Y$ and $Y^{\prime}$.

Lemma A.1. Let $f: X \rightarrow X^{\prime}$ and $g: X^{\prime} \rightarrow X^{\prime \prime}$ be A-equivariant proper morphisms of A-varieties. Then we have

$$
(g \circ f)_{*}=g_{*} \circ f_{*}: K^{A}(X) \longrightarrow K^{A}\left(X^{\prime \prime}\right) .
$$

Assume that we are given an $A$-equivariant morphism $f: Y \rightarrow Y^{\prime}$ of $A$-varieties and an $A$-stable closed subvariety $X^{\prime}$ of $Y^{\prime}$. Set $X=f^{-1}\left(X^{\prime}\right)$. If $f$ is smooth or if $Y^{\prime}$ is a smooth variety, then the derived functors

$$
L^{n} f^{*}: \operatorname{Coh}^{A}\left(Y^{\prime} ; X^{\prime}\right) \longrightarrow \operatorname{Coh}^{A}(Y ; X) \quad(n \in \mathbb{Z})
$$

of the inverse image functor

$$
f^{*}: \operatorname{Coh}^{A}\left(Y^{\prime} ; X^{\prime}\right) \longrightarrow \operatorname{Coh}^{A}(Y ; X) \quad\left(M \longmapsto \mathcal{O}_{Y} \otimes_{f^{-1} \mathcal{O}_{Y^{\prime}}} f^{-1} M\right)
$$

are zero except for finitely many $n$ 's, and they induce a homomorphism

$$
\text { (A.3) } f^{*}: K^{A}\left(Y^{\prime} ; X^{\prime}\right) \longrightarrow K^{A}(Y ; X) \quad\left([M] \longmapsto \sum_{n}(-1)^{n}\left[L^{n} f^{*}(M)\right]\right)
$$

of $R^{A}$-modules. If $f$ is smooth, we have $L^{n} f^{*}=0$ for $n \neq 0$.

Lemma A.2. Let $f: Y \rightarrow Y^{\prime}$ and $g: Y^{\prime} \rightarrow Y^{\prime \prime}$ be A-equivariant morphisms of A-varieties. Let $X^{\prime \prime}$ be a closed subvariety of $Y^{\prime \prime}$, and set $X=(g \circ f)^{-1}\left(X^{\prime \prime}\right), X^{\prime}=f^{-1}\left(X^{\prime \prime}\right)$. Assume that $f^{*}: K^{A}\left(Y^{\prime} ; X^{\prime}\right) \rightarrow$ $K^{A}(Y ; X)$ and $g^{*}: K^{A}\left(Y^{\prime \prime} ; X^{\prime \prime}\right) \rightarrow K^{A}\left(Y^{\prime} ; X^{\prime}\right)$ are defined. Then we have

$$
(g \circ f)^{*}=f^{*} \circ g^{*}: K^{A}\left(Y^{\prime \prime} ; X^{\prime \prime}\right) \longrightarrow K^{A}(Y ; X) .
$$


Assume that we are given a smooth $A$-variety $Y$ and its $A$-stable closed subvarieties $X_{1}$ and $X_{2}$. The derived functors

$$
\begin{gathered}
\operatorname{Tor}_{n}^{\mathcal{O}_{Y}}(,): \operatorname{Coh}^{A}\left(Y ; X_{1}\right) \times \operatorname{Coh}^{A}\left(Y ; X_{2}\right) \longrightarrow \operatorname{Coh}^{A}\left(Y ; X_{1} \cap X_{2}\right) \\
\left(\left(M_{1}, M_{2}\right) \longmapsto \operatorname{Tor}_{n}^{\mathcal{O}_{Y}}\left(M_{1}, M_{2}\right)=H^{-n}\left(M_{1} \otimes \mathcal{O}_{Y}^{\mathbb{L}} M_{2}\right)\right)
\end{gathered}
$$

of the tensor product functor $\otimes_{\mathcal{O}_{Y}}$ are zero except for finitely many $n$ 's, and induce a bilinear map

$$
\begin{aligned}
\otimes_{\mathcal{O}_{Y}}: K^{A}\left(Y ; X_{1}\right) \times K^{A}\left(Y ; X_{2}\right) & \longrightarrow K^{A}\left(Y ; X_{1} \cap X_{2}\right) \\
\left(\left(\left[M_{1}\right],\left[M_{2}\right]\right)\right. & \left.\longmapsto\left[M_{1}\right] \otimes_{\mathcal{O}_{Y}}\left[M_{2}\right]=\sum_{n}(-1)^{n} \operatorname{Tor}_{n}^{\mathcal{O}_{Y}}\left(M_{1}, M_{2}\right)\right)
\end{aligned}
$$

of $R^{A}$-modules. Note that $\otimes \mathcal{O}_{Y}$ does depend on the choice of the ambient space $Y$.

Lemma A.3. Let $f: Y \rightarrow Y^{\prime}$ be an A-equivariant smooth morphism of smooth A-varieties. Let $X_{1}^{\prime}, X_{2}^{\prime}$ be closed subvarieties of $Y^{\prime}$, and set $X_{1}=f^{-1}\left(X_{1}^{\prime}\right), X_{2}=f^{-1}\left(X_{2}^{\prime}\right)$. Then we have

$$
f^{*}\left(m_{1}\right) \otimes \mathcal{O}_{Y} f^{*}\left(m_{2}\right)=f^{*}\left(m_{1} \otimes_{\mathcal{O}_{Y^{\prime}}} m_{2}\right) \in K^{A}\left(Y ; X_{1} \cap X_{2}\right)
$$

for any $m_{1} \in K^{A}\left(Y^{\prime} ; X_{1}^{\prime}\right), m_{2} \in K^{A}\left(Y^{\prime} ; X_{2}^{\prime}\right)$.

Lemma A.4. (Projection formula) Let $f: Y \rightarrow Y^{\prime}$ be an A-equivariant morphism of smooth A-varieties. Let $X_{1}^{\prime}$ be an A-stable closed subvariety of $Y^{\prime}$ and set $X_{1}=f^{-1}\left(X_{1}^{\prime}\right)$. Let $X_{2}$ and $X_{2}^{\prime}$ be closed subvarieties of $Y$ and $Y^{\prime}$ respectively such that $f\left(X_{2}\right)=X_{2}^{\prime}$ and $X_{2} \rightarrow X_{2}^{\prime}$ is proper. Then we have

$$
f_{*}\left(f^{*}(m) \otimes_{\mathcal{O}_{Y}} n\right)=m \otimes \mathcal{O}_{Y^{\prime}} f_{*} n \in K^{A}\left(Y^{\prime} ; X_{1}^{\prime} \cap X_{2}^{\prime}\right)
$$

for any $m \in K^{A}\left(Y^{\prime} ; X_{1}^{\prime}\right), n \in K^{A}\left(Y ; X_{2}\right)$.

LEmma A.5. (Base change theorem 1) Let $f: Y^{\prime} \rightarrow Y$ and $g: Y^{\prime \prime} \rightarrow Y$ be A-equivariant morphism of A-varieties. We assume that $g$ is smooth. Set $Y^{\prime \prime \prime}=Y^{\prime} \times_{Y} Y^{\prime \prime}$ and let $f^{\prime}: Y^{\prime \prime \prime} \rightarrow Y^{\prime \prime}$ and $g^{\prime}: Y^{\prime \prime \prime} \rightarrow Y^{\prime}$ be canonical morphisms. Let $X, X^{\prime}$ be closed A-stable closed subvarieties of $Y, Y^{\prime}$ respectively such that $f\left(X^{\prime}\right) \subset X$ and $X^{\prime} \rightarrow X$ is proper. Then we have

$$
g^{*} \circ f_{*}=f_{*}^{\prime} \circ g^{\prime *}: K^{A}\left(Y^{\prime} ; X^{\prime}\right) \longrightarrow K^{A}\left(Y^{\prime \prime} ; g^{-1}(X)\right) .
$$


Lemma A.6. (Base change theorem 2) Let $Y$ be a smooth A-variety and let $Y_{1}, Y_{2}$ be A-stable smooth closed subvarieties of $Y$. Set $Y_{3}=Y_{1} \cap Y_{2}$. We assume that $Y_{3}$ is smooth and that

$$
T_{y} Y=T_{y} Y_{1}+T_{y} Y_{2}, \quad T_{y} Y_{3}=T_{y} Y_{1} \cap T_{y} Y_{2}
$$

for any $y \in Y_{3}$. Here, $T_{y} Y$ denotes the tangent space of $Y$ at $y$. Let $i: Y_{1} \rightarrow Y, j: Y_{2} \rightarrow Y, i^{\prime}: Y_{3} \rightarrow Y_{2}, j^{\prime}: Y_{3} \rightarrow Y_{1}$ be the inclusions. Let $X_{1}$ be an A-stable closed subvariety of $Y_{1}$. Then we have

$$
j^{*} \circ i_{*}=i_{*}^{\prime} \circ j^{\prime *}: K^{A}\left(Y_{1} ; X_{1}\right) \longrightarrow K^{A}\left(Y_{2} ; X_{1} \cap Y_{2}\right) .
$$

\section{Appendix B. Convolution product}

In this section $G$ is as in Section 2. In particular, $G$ is not necessarily of type $A$. We fix a nilpotent orbit $O$ of $\mathfrak{g}$ in the following.

According to Conjecture 3.5 the quotient

$$
H\left(\tilde{W}_{a}\right)_{\mathcal{C}_{O}}=H\left(\tilde{W}_{a}\right)_{L R}^{\leq \mathcal{C}_{O}} / H\left(\tilde{W}_{a}\right)_{L R} \mathcal{C}_{O}
$$

should be identified with

$$
K^{G \times \mathbb{C}^{*}}\left(Z_{O}\right) \cong K^{G \times \mathbb{C}^{*}}\left(Z_{\bar{O}}\right) / K^{G \times \mathbb{C}^{*}}\left(Z_{\bar{O} \backslash O}\right) .
$$

For $e \in O$ set

$$
\mathcal{B}_{e}=\left\{x \in \mathcal{B} \mid e \in \mathfrak{n}_{x}\right\} .
$$

Since $Z_{O}$ is a $G \times \mathbb{C}^{*}$-equivariant fiber bundle on $O$ whose fiber at $e \in O$ is canonically isomorphic to $\mathcal{B}_{e} \times \mathcal{B}_{e}$, we have

$$
K^{G \times \mathbb{C}^{*}}\left(Z_{O}\right) \cong K^{M(e)}\left(\mathcal{B}_{e} \times \mathcal{B}_{e}\right),
$$

where

$$
M(e)=\left\{(g, z) \in G \times \mathbb{C}^{*} \mid \operatorname{Ad}(g)(e)=z^{2} e\right\} .
$$

The aim of this section is to give a description of the product on $K^{M(e)}\left(\mathcal{B}_{e} \times \mathcal{B}_{e}\right)$ induced from the convolution product $\star$ on $K^{G \times \mathbb{C}^{*}}(Z)$.

We say that a triple $(h, e, f) \in \mathfrak{g} \times \mathfrak{g} \times \mathfrak{g}$ is an $\mathfrak{s l}_{2}$-triple if $[h, e]=2 e$, $[h, f]=-2 f,[e, f]=h$. Then $e$ and $f$ are nilpotent elements belonging to the same conjugacy class. Moreover, the map $(h, e, f) \mapsto e$ induces a bijection between the set of $G$-conjugacy classes of $\mathfrak{s l}_{2}$-triples and that of nilpotent orbits. Set

$$
\hat{O}=\left\{(e, f) \in \mathfrak{g} \times \mathfrak{g} \mid e \in O,([e, f], e, f) \text { is an } \mathfrak{s l}_{2} \text {-triple }\right\} .
$$


The group $G \times \mathbb{C}^{*}$ acts transitively on $\hat{O}$ by

$$
(g, z):(e, f) \longrightarrow\left(z^{-2} \operatorname{Ad}(g)(e), z^{2} \operatorname{Ad}(g)(f)\right) .
$$

In particular, $\hat{O}$ is a smooth variety. For $(e, f) \in \hat{O}$, Slodowy's variety $\Lambda_{(e, f)}$ is defined by

$$
\Lambda_{(e, f)}=\{(a, x) \in \Lambda \mid a \in e+\mathfrak{z} \mathfrak{g}(f)\}
$$

where

$$
\mathfrak{z} \mathfrak{g}(f)=\{a \in \mathfrak{g} \mid[a, f]=0\} .
$$

Proposition B.1. (Slodowy [17])

(i) $\Lambda_{(e, f)}$ is a smooth variety with $\operatorname{dim} \Lambda_{(e, f)}=2 \operatorname{dim} \mathcal{B}_{e}$.

(ii) $\operatorname{Ad}(G)(\mathcal{N} \cap(e+\mathfrak{z} \mathfrak{g}(f)) \subset \mathcal{N} \backslash(\bar{O} \backslash O)$.

(iii) $O \cap(e+\mathfrak{z} \mathfrak{g}(f))=\{e\}$.

We identify $\mathcal{B}_{e}$ with a closed subvariety of $\Lambda_{(e, f)}$ via the embedding $x \mapsto(e, x)$. Set

$$
M(e, f)=\left\{(g, z) \in G \times \mathbb{C}^{*} \mid \operatorname{Ad}(g)(e)=z^{2} e, \operatorname{Ad}(g)(f)=z^{-2} f\right\} .
$$

Then $M(e, f)$ is a subgroup of $M(e)$ acting naturally on $\Lambda_{(e, f)}$. Moreover, $M(e, f)$ and $M(e)$ contain a common maximal reductive subgroup (see [6]). Hence we have the identification

$$
K^{M(e)}\left(\mathcal{B}_{e} \times \mathcal{B}_{e}\right)=K^{M(e, f)}\left(\Lambda_{(e, f)} \times \Lambda_{(e, f)} ; \mathcal{B}_{e} \times \mathcal{B}_{e}\right)
$$

For $(i, j)=(1,2),(2,3),(1,3)$ we denote by $\pi_{i j}: \Lambda_{(e, f)} \times \Lambda_{(e, f)} \times \Lambda_{(e, f)} \rightarrow$ $\Lambda_{(e, f)} \times \Lambda_{(e, f)}$ the projections onto $(i, j)$-factors.

Theorem B.2. The product on $K^{M(e, f)}\left(\Lambda_{(e, f)} \times \Lambda_{(e, f)} ; \mathcal{B}_{e} \times \mathcal{B}_{e}\right)$ induced from the convolution product $\star$ on $K^{G \times \mathbb{C}^{*}}(Z)$ is given by

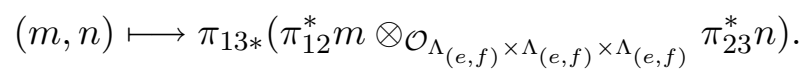

The rest of this section is devoted to proving Theorem B.2.

Set

$$
\tilde{\Lambda}=\{(a, x) \in \Lambda \mid a \notin \bar{O} \backslash O\} .
$$

Then $\tilde{\Lambda}$ is an open subset of $\Lambda$, and $Z_{O}$ is a closed subset of $\tilde{\Lambda} \times \tilde{\Lambda}$. We denote by

$$
k: Z_{O} \longrightarrow \tilde{\Lambda} \times \tilde{\Lambda}
$$


the closed embedding. For $(i, j)=(1,2),(2,3),(1,3)$ we denote by $\tilde{p}_{i j}$ : $\tilde{\Lambda} \times \tilde{\Lambda} \times \tilde{\Lambda} \rightarrow \tilde{\Lambda} \times \tilde{\Lambda}$ the projections onto $(i, j)$-factors. We see easily the following.

Lemma B.3. The product on $K^{G \times \mathbb{C}^{*}}\left(Z_{O}\right)=K^{G \times \mathbb{C}^{*}}\left(\tilde{\Lambda} \times \tilde{\Lambda} ; Z_{O}\right)$ induced from the convolution product $\star$ on $K^{G \times \mathbb{C}^{*}}(Z)$ is given by

$$
(m, n) \longmapsto m \star n=\tilde{p}_{13 *}\left(\tilde{p}_{12}^{*} m \otimes_{\mathcal{O}_{\tilde{\Lambda} \times \tilde{\Lambda} \times \tilde{\Lambda}}} \tilde{p}_{23}^{*} n\right) .
$$

Set

$$
\begin{aligned}
\tilde{\Lambda}_{O} & =\{(e, x) \in \Lambda \mid e \in O\}, \\
Y_{O} & =\tilde{\Lambda}_{O} \times{ }_{O} \hat{O}=\{(e, f, x) \mid(e, f) \in \hat{O},(e, x) \in \Lambda\} \\
Y & =\left\{(e, f, a, x) \mid(e, f) \in \hat{O},(a, x) \in \Lambda_{(e, f)}\right\} .
\end{aligned}
$$

We identify $Y_{O}$ with a closed subvariety of $Y$ by the embedding

$$
i: Y_{O} \longrightarrow Y \quad((e, f, x) \longmapsto(e, f, e, x)) .
$$

Then $Y$ is a $G \times \mathbb{C}^{*}$-equivariant fiber bundle on $\hat{O}$ whose fiber at $(e, f) \in \hat{O}$ is $\Lambda_{(e, f)}$, and $Y_{O}$ is its subbundle whose fiber at $(e, f) \in \hat{O}$ is $\mathcal{B}_{e}$. In particular, $Y$ is a smooth variety and the projection $Y \rightarrow \hat{O}$ is a smooth morphism.

We set

$$
\begin{aligned}
Y^{(2)} & =Y \times_{\hat{O}} Y \\
& =\left\{(e, f, a, x, b, y) \mid(e, f) \in \hat{O},(a, x),(b, y) \in \Lambda_{(e, f)}\right\}, \\
Y_{O}^{(2)} & =Y_{O} \times_{\hat{O}} Y_{O}=Z_{O} \times_{O} \hat{O} \\
& =\left\{(e, f, x, y) \mid(e, f) \in \hat{O}, x, y \in \mathcal{B}_{e}\right\} .
\end{aligned}
$$

We regard $Y_{O}^{(2)}$ as a closed subvariety of $Y^{(2)}$ by the embedding

$$
i^{(2)}=i \times_{\hat{O}} i: Y_{O}^{(2)} \longrightarrow Y^{(2)} .
$$

Define

$$
\varphi: Y_{O}^{(2)} \longrightarrow Z_{O}
$$

by $\varphi(e, f, x, y)=(e, x, y)$. It is a smooth surjective morphism. Since $Y_{O}^{(2)}$ is a $G \times \mathbb{C}^{*}$-equivariant fiber bundle whose fiber at $(e, f) \in \hat{O}$ is $\Lambda_{(e, f)} \times \Lambda_{(e, f)}$, 
we have a commutative diagram

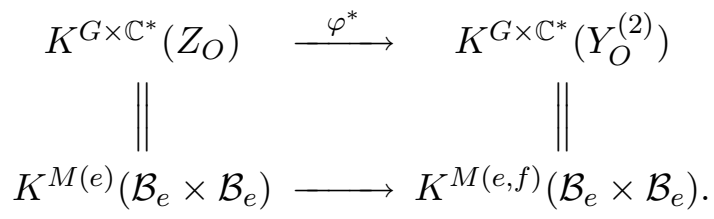

Hence we see by (B.2) that

$$
\varphi^{*}: K^{G \times \mathbb{C}^{*}}\left(Z_{O}\right) \longrightarrow K^{G \times \mathbb{C}^{*}}\left(Y_{O}^{(2)}\right)
$$

is an isomorphism of $R^{G \times \mathbb{C}^{*}}$-modules. Set

$$
\begin{aligned}
& Y^{(3)}=Y \times_{\hat{O}} Y \times_{\hat{O}} Y, \\
& Y_{O}^{(3)}=Y_{O} \times_{\hat{O}} Y \times_{\hat{O}} Y,
\end{aligned}
$$

and regard $Y_{O}^{(3)}$ as a subvariety of $Y^{(3)}$ by

$$
i^{(3)}=i \times_{\hat{O}} i \times_{\hat{O}} i: Y_{O}^{(3)} \longrightarrow Y^{(3)} .
$$

For $(i, j)=(1,2),(2,3),(1,3)$ we denote by $q_{i j}: Y^{(3)} \rightarrow Y^{(2)}$ the projections onto $(i, j)$-factors. Note that $q_{i j}$ is a morphism of $G \times \mathbb{C}^{*}$-equivariant fiber bundles on $\hat{O}$ whose fiber at $(e, f) \in \hat{O}$ is given by $\pi_{i j}: \Lambda_{(e, f)} \times$ $\Lambda_{(e, f)} \times \Lambda_{(e, f)} \rightarrow \Lambda_{(e, f)} \times \Lambda_{(e, f)}$. Therefore, Theorem B.2 is equivalent to the following.

Proposition B.4. The product on $K^{G \times \mathbb{C}^{*}}\left(Y^{(2)} ; Y_{O}^{(2)}\right)$ induced from the convolution product $\star$ on $K^{G \times \mathbb{C}^{*}}\left(Z_{O}\right)$ via $\varphi^{*}$ is given by

$$
(m, n) \longmapsto q_{13 *}\left(q_{12}^{*} m \otimes_{\mathcal{O}_{Y^{(3)}}} q_{23}^{*} n\right) .
$$

By Proposition B.1 we have a morphism

$$
\theta: Y \longrightarrow \tilde{\Lambda} \quad((e, f, a, x) \longmapsto(a, x))
$$

We define

$$
\tau: Y_{O} \longrightarrow \tilde{\Lambda}_{O}
$$

as the restriction of $\theta$. 
Lemma B.5. (i) The commutative diagram

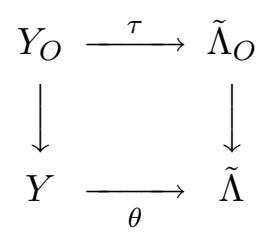

is cartesian.

(ii) $\theta$ is a smooth morphism.

Proof. The statement (i) follows from Proposition B.1 (iii). By a result of Slodowy [17] we see that the composition of the smooth surjective morphism

$$
G \times \Lambda_{(e, f)} \longrightarrow Y \quad((g,(a, x)) \longmapsto(\operatorname{Ad}(g)(e), \operatorname{Ad}(g)(f), \operatorname{Ad}(g)(a), g x))
$$

with $\theta: Y \rightarrow \tilde{\Lambda}$ is smooth (see the proof of Proposition 11.10 in Lusztig $[13])$. Hence $\theta$ is also smooth.

Consider the following diagrams

$$
\begin{aligned}
& Y_{O}^{(2)} \times_{\hat{O}} Y \stackrel{i^{(2)} \times_{\hat{O}}}{\longrightarrow} \quad Y^{(3)} \stackrel{k_{23}}{\longrightarrow} \tilde{\Lambda} \times Y^{(2)} \\
& Y_{O}^{(2)} \times \tilde{\Lambda} \underset{i^{(2)} \times 1}{\longrightarrow} Y^{(2)} \times \tilde{\Lambda} \underset{\ell_{12}}{\longrightarrow} \tilde{\Lambda} \times Y \times \tilde{\Lambda}
\end{aligned}
$$

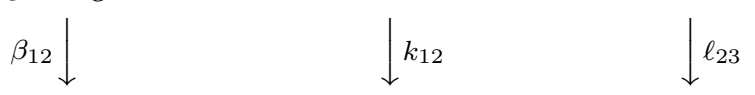

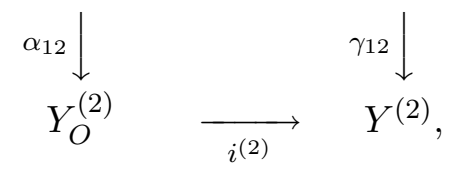

$$
\begin{array}{ccc}
Y_{O}^{(2)} \times \tilde{\Lambda} & \stackrel{\ell_{12} \circ\left(i^{(2)} \times 1\right)}{\longrightarrow} \tilde{\Lambda} \times Y \times \tilde{\Lambda} \\
\varphi \circ \alpha_{12} \downarrow & & \\
Z_{O} & \longrightarrow \tilde{p}_{12} \circ(1 \times \theta \times 1) \\
& & \tilde{\Lambda} \times \tilde{\Lambda}
\end{array}
$$

where $\alpha_{12}, \gamma_{12}$ are the projections, and $\beta_{12}, k_{12}, k_{23}, \ell_{12}, \ell_{23}$ are the closed embeddings induced by $\theta: Y \rightarrow \tilde{\Lambda}$. We can check the commutativity easily. 
Moreover, we see easily that all of the squares in the diagrams are cartesian. We set

$$
\psi=\ell_{12} \circ k_{12}=\ell_{23} \circ k_{23}: Y^{(3)} \longrightarrow \tilde{\Lambda} \times Y \times \tilde{\Lambda} .
$$

Let $m, n \in K^{G \times \mathbb{C}^{*}}\left(Z_{O} ; Z_{O}\right)$. Then the corresponding elements in $K^{G \times \mathbb{C}^{*}}\left(Y^{(2)} ; Y_{O}^{(2)}\right)$ are given by $\tilde{m}=i_{*}^{(2)} \varphi^{*} m, \tilde{n}=i_{*}^{(2)} \varphi^{*} n$ respectively. By $q_{12}=\gamma_{12} \circ k_{12}$ we see from (B.4) that

$$
q_{12}^{*} \tilde{m}=k_{12}^{*} \gamma_{12}^{*} i_{*}^{(2)} \varphi^{*} m=k_{12}^{*}\left(i^{(2)} \times 1\right)_{*} \alpha_{12}^{*} \varphi^{*} m .
$$

Similarly, we have $q_{23}^{*} \tilde{n}=k_{23}^{*}\left(1 \times i^{(2)}\right)_{*} \alpha_{23}^{*} \varphi^{*} n$, where $\alpha_{23}: \tilde{\Lambda} \times Y_{O}^{(2)} \rightarrow Y_{O}^{(2)}$ is the projection. Hence we have

$$
\begin{aligned}
\psi_{*}\left(q_{12}^{*} \tilde{m} \otimes q_{23}^{*} \tilde{n}\right) & =\ell_{12 *} k_{12 *}\left(k_{12}^{*}\left(i^{(2)} \times 1\right)_{*} \alpha_{12}^{*} \varphi^{*} m \otimes k_{23}^{*}\left(1 \times i^{(2)}\right)_{*} \alpha_{23}^{*} \varphi^{*} n\right) \\
& =\ell_{12 *}\left(\left(i^{(2)} \times 1\right)_{*} \alpha_{12}^{*} \varphi^{*} m \otimes k_{12 *} k_{23}^{*}\left(1 \times i^{(2)}\right)_{*} \alpha_{23}^{*} \varphi^{*} n\right) \\
& =\ell_{12 *}\left(\left(i^{(2)} \times 1\right)_{*} \alpha_{12}^{*} \varphi^{*} m \otimes \ell_{12}^{*} \ell_{23 *}\left(1 \times i^{(2)}\right)_{*} \alpha_{23}^{*} \varphi^{*} n\right) \\
& =\ell_{12 *}\left(i^{(2)} \times 1\right)_{*} \alpha_{12}^{*} \varphi^{*} m \otimes \ell_{23 *}\left(1 \times i^{(2)}\right)_{*} \alpha_{23}^{*} \varphi^{*} n .
\end{aligned}
$$

Here we have used Lemma A.4 for the second and the fourth identities and Lemma A.6 for the third identity. By Lemma A.5, Lemma B.5 and (B.5) we have

$$
\ell_{12 *}\left(i^{(2)} \times 1\right)_{*} \alpha_{12}^{*} \varphi^{*} m=(1 \times \theta \times 1)^{*} \tilde{p}_{12}^{*} k_{*} m .
$$

Similarly we have

$$
\ell_{23_{*}}\left(1 \times i^{(2)}\right)_{*} \alpha_{23}^{*} \varphi^{*} n=(1 \times \theta \times 1)^{*} \tilde{p}_{23}^{*} k_{*} n .
$$

Therefore, we obtain

$$
\psi_{*}\left(q_{12}^{*} \tilde{m} \otimes q_{23}^{*} \tilde{n}\right)=(1 \times \theta \times 1)^{*}\left(\tilde{p}_{12}^{*} k_{*} m \otimes \tilde{p}_{23}^{*} k_{*} n\right)
$$

by Lemma A.3.

Set

$$
\tilde{\Lambda}_{O}^{(3)}=\tilde{\Lambda}_{O} \times_{O} \tilde{\Lambda}_{O} \times_{O} \tilde{\Lambda}_{O}=\tilde{p}_{12}^{-1} Z_{O} \cap \tilde{p}_{23}^{-1} Z_{O},
$$

and consider the commutative diagram

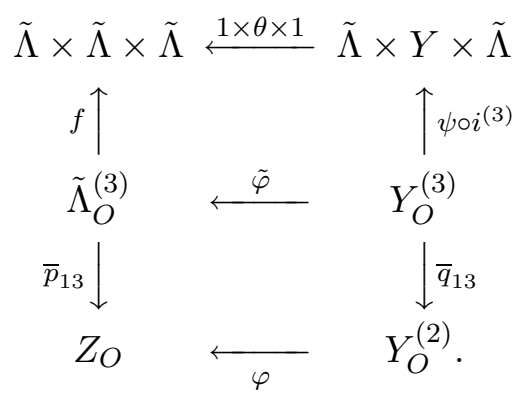


Here, $f$ is the natural inclusion, $\tilde{\varphi}$ is the canonical morphism, and $\bar{p}_{13}, \bar{q}_{13}$ are the restrictions of $\tilde{p}_{13}, q_{13}$ respectively. We see easily that both of the squares in (B.6) are cartesian.

Define $u \in K^{G \times \mathbb{C}^{*}}\left(\tilde{\Lambda}_{O}^{(3)} ; \tilde{\Lambda}_{O}^{(3)}\right)$ by $f_{*} u=\tilde{p}_{12}^{*} k_{*} m \otimes \tilde{p}_{23}^{*} k_{*} n$. Then we have

$$
\psi_{*}\left(q_{12}^{*} \tilde{m} \otimes q_{23}^{*} \tilde{n}\right)=(1 \times \theta \times 1)^{*} f_{*} u=\psi_{*}\left(i_{*}^{(3)} \tilde{\varphi}^{*} u\right)
$$

and hence $q_{12}^{*} \tilde{m} \otimes q_{23}^{*} \tilde{n}=i_{*}^{(3)} \tilde{\varphi}^{*} u$. It follows that

$$
q_{13 *}\left(q_{12}^{*} \tilde{m} \otimes q_{23}^{*} \tilde{n}\right)=q_{13 *} i_{*}^{(3)} \tilde{\varphi}^{*} u=i_{*}^{(2)} \bar{q}_{13 *} \tilde{\varphi}^{*} u=i_{*}^{(2)} \varphi^{*}\left(\bar{p}_{13 *} u\right) .
$$

By

$$
k_{*}\left(\bar{p}_{13 *} u\right)=\tilde{p}_{13 *} f_{*} u=\tilde{p}_{13 *}\left(\tilde{p}_{12}^{*} k_{*} m \otimes \tilde{p}_{23}^{*} k_{*} n\right)
$$

we conclude that the element of $K^{G \times \mathbb{C}^{*}}\left(Y^{(2)} ; Y_{O}^{(2)}\right)$ corresponding to $m$ $n \in K^{G \times \mathbb{C}^{*}}\left(Z_{O} ; Z_{O}\right)$ is given by $q_{13 *}\left(q_{12}^{*} \tilde{m} \otimes q_{23}^{*} \tilde{n}\right)$. Proposition B.4 is verified. This completes the proof of Theorem B.2.

Acknowledgment. The work started during TT's fruitful visit to the Chinese Academy of Science in 2001, and was completed during NX's enjoyable visit to the Department of Mathematics, Osaka City University in 2004 under the financial support of the 21-st century COE program "Constitution of wide-angle mathematical basis focused on knots". TT and NX would like to thank the hospitality of those institutions. TT and NX are grateful to the referee for helpful comments.

\section{REFERENCES}

[1] R. Bezrukavnikov, Perverse sheaves on affine flags and nilpotent cone of the Langlands dual group, math.RT/0201256.

[2] N. Chriss and V. Ginzburg, Representation theory and complex geometry, Birkhäuser Boston, Inc., Boston, MA, 1997.

[3] V. Ginzburg, Lagrangian construction of representations of Hecke algebras, Adv. in Math., 63 (1987), 100-112.

[4] V. Ginzburg, Geometrical aspects of representation theory, Proceedings of the International Congress of Mathematicians, Vol. 1, 2 (Berkeley, Calif., 1986), Providence, RI (1987), pp. 840-848.

[5] D. Kazhdan and G. Lusztig, Representations of Coxeter groups and Hecke algebras, Invent. Math., 53 (1979), 165-184.

[6] D. Kazhdan and G. Lusztig, Proof of the Deligne-Langlands conjecture for Hecke algebras, Invent. Math., 87 (1987), 153-215. 
[7] G. Lusztig, Some problems in the representation theory of finite Chevalley groups, The Santa Cruz Conference on Finite Groups (Univ. California, Santa Cruz, Calif., 1979), Proc. Sympos. Pure Math., 37, Amer. Math. Soc., Providence, R.I. (1980), pp. 313-317.

[8] G. Lusztig, Some examples of square integrable representations of semisimple p-adic groups, Trans. Amer. Math. Soc., 277 (1983), 623-653.

[9] G. Lusztig, The two-sided cells of the affine Weyl group of type $A_{n}$, Infinite-dimensional groups with applications (Berkeley, Calif., 1984), Math. Sci. Res. Inst. Publ., 4, Springer, New York (1985), pp. 275-283.

[10] G. Lusztig, Cells in affine Weyl groups, Algebraic groups and related topics, Advanced Studies in Pure Math., vol. 6, Kinokuniya and North Holland (1985), pp. 255-287.

[11] G. Lusztig, Cells in affine Weyl groups, II, J. Alg., 109 (1987), 536-548.

[12] G. Lusztig, Cells in affine Weyl groups, IV, J. Fac. Sci. Univ. Tokyo Sect. IA Math., 36 (1989), 297-328.

[13] G. Lusztig, Bases in equivariant K-theory, Represent. Theory, 2 (1998), 298-369 (electronic).

[14] G. Lusztig, Bases in equivariant K-theory, II, Represent. Theory, 3 (1999), 281-353 (electronic).

[15] J.-Y. Shi, The Kazhdan-Lusztig cells in certain affine Weyl groups, Lecture Notes in Mathematics 1179, Springer-Verlag, Berlin, 1986.

[16] J.-Y. Shi, The partial order on two-sided cells of certain affine Weyl groups, J. Algebra, 179 (1996), 607-621.

[17] P. Slodowy, Simple singularities and simple algebraic groups, Lecture Notes in Mathematics 815, Springer, Berlin, 1980.

[18] T. Tanisaki, Hodge modules, equivariant $K$-theory and Hecke algebras, Publ. Res. Inst. Math. Sci., 23 (1987), 841-879.

[19] T. Tanisaki, Representations of semisimple Lie groups and D-modules, Sugaku expositions, 4 (1991), 43-61.

[20] R. W. Thomason, Equivariant algebraic versus topological K-homology Atiyah-Segal style, Duke Math. J., 56 (1988), 589-636.

[21] N. Xi, Representations of affine Hecke algebras, Lecture Notes in Mathematics 1587, Springer-Verlag, Berlin, 1994.

[22] N. Xi, The based ring of two-sided cells of affine Weyl groups of type $\tilde{A}_{n-1}$, Mem. of AMS, Vol. 157, No. 749, 2002.

Toshiyuki Tanisaki

Department of Mathematics

Osaka City University

3-3-138 Sugimoto

Sumiyoshi-ku

Osaka, 558-8585

Japan

tanisaki@sci.osaka-cu.ac.jp 
Nanhua Xi

Institute of Mathematics

Chinese Academy of Sciences

Beijing, 100080

China

nanhua@math.ac.cn 